\title{
Polarization Transport of Transverse Acoustic Waves: Berry Phase and Spin Hall Effect of Phonons
}

\author{
K. Yu. Bliokh ${ }^{1,2,3}$ and V. D. Freilikher ${ }^{3}$ \\ ${ }^{1}$ Institute of Radio Astronomy, 4 Krasnoznamyonnaya St., Kharkov 61002, Ukraine \\ ${ }^{2}$ A.Ya. Usikov Institute of Radiophysics and Electronics, 12 Akademika Proskury St., \\ Kharkov 61085, Ukraine \\ ${ }^{3}$ Department of Physics, Bar-Ilan University, Ramat Gan 52900, Israel
}

\section{Published as}

K. Yu. Bliokh and V. D. Freilikher,

Phys. Rev. B 74, 174302 (2006).
and technology. 